\title{
Fractal and Fracture Mechanics Analyses on Fatigue Fracture Surfaces of Metallic Materials
}

\author{
Takashi SAKAI $^{1}$, Tatsuo SAKAI ${ }^{2}$ and Akira UENO ${ }^{3}$ \\ ${ }^{1}$ Department of Mechanical Engineering, Osaka Institute of Technology \\ 5-16-1 Omiya, Asahi-ku, Osaka, 535-8585 JAPAN \\ ${ }^{2}$ Faculty of Science and Engineering, Ritsumeikan University \\ 1-1-1 Nojihigashi, Kusatsu, Shiga, 525-8577 JAPAN \\ ${ }^{3}$ Department of Mechanical System Engineering, Toyota Institute of Technology \\ 2-12-1 Hisakata, Tempaku-ku, Nagoya, Aichi, 468-8511 JAPAN
}

\begin{abstract}
In this study, fatigue crack propagation tests were carried out to obtain the fatigue fracture surfaces on compact tension type specimens of A5052 aluminum alloy and S25C carbon steel, and the fatigue fracture surfaces were observed by means of a scanning laser microscope system. Based on the digital data thus obtained, imaginary fracture surface was reconstructed in a 3-dimensional space by a personal computer. Fractal analysis proposed by B. B. Mandelbrot was applied to such 3-dimensional surfaces and a hyperbola model was accepted to represent the Richardson effect. Due to the Richardson effect thus analysed, the fractal feature was confirmed in the fracture surface irregularity. It was finally found that the geometrical irregularity of the surface was well evaluated by combining the fractal dimension and additional indices termed as "index of fracture surface nature", and that the fractal dimension and the additional indices were successfully connected to the stress intensity factor range of $\Delta K$.
\end{abstract}

\section{KEYWORDS}

Fractal, Richardson Effect, Self-similarity, Fatigue Fracture Surface, Surface Irregularity, Fractography, Fracture Mechanics, SLM Observation

\section{INTRODUCTION}

In order to analyse the irregularity of shapes and time-dependent phenomena, various methods such as Fourier analysis and some conventional procedures as stochastic process have been developed and used by many researchers. A concept of fractal proposed by B. B. Mandelbrot [1] is useful to quantify the above irregularity and this has been successfully applied to various fields in both science and technology [2-5].

On the other hand, several kinds of high resolution microscopes such as SLM (Scanning Laser Microscope), STM (Scanning Tunnel Microscope) and AFM (Atomic Force Microscope) have been used in recent years to observe the nature of the fracture surface in a 3-dimensional space. However, definite procedures to quantify the irregularity of the fracture surfaces have not been established yet.

As reported in the earlier papers [6,7], we proposed a new analytical method to quantify the surface irregularity applying the concept of fractal, and the surface irregularity of the tensile fracture surfaces and the mechanically finished surfaces was successfully evaluated by using this method. In the present study, this method is modified and expanded to analyse the surface irregularity of the fatigue fracture surfaces. Moreover, particular attention is paid here to investigate the relationship between the surface irregularity 
and the fracture mechanics parameter of $\Delta K$.

\section{SPECIMENS AND FATIGUE CRACK PROPAGATION TESTS}

\subsection{Specimens and Fatigue Crack Propagation Behavior}

Materials used in this study are A5052 aluminum alloy and S25C carbon steel in JIS material codes. Mechanical properties of these materials obtained by tensile tests are listed in Table 1. Fatigue crack propagation tests were performed by a hydraulic servo fatigue testing machine to obtain the fatigue fracture surfaces on compact tension type specimen $[8,9]$ standardized in ASTM. The frequency was fixed to $50 \mathrm{~Hz}$, and two different stress ratios of $R=0.1$ and 0.5 were selected. Fatigue fracture surfaces were observed by means of a SLM system explained in other papers [10, 11], whereas the fatigue crack propagation was observed by an optical microscope with the resolution of $\times 100$.

Based on the crack propagation behavior thus observed, relationships between the stress intensity factor range $\Delta K$ and the crack growth rate $d a / d N$ are plotted as shown in Figure 1. It should be noted that only one specimen was assigned to each series of the fatigue crack propagation test. In this figure, each solid line is determined as to provide the least squares for the respective data points. It is confirmed that the fatigue crack propagation behavior is well represented by the following expression [12];

$$
\frac{d a}{d N}=C(\Delta K)^{m}
$$

where

$$
\begin{gathered}
\Delta K=\frac{\Delta P}{B W^{\frac{1}{2}}} \times f(\alpha), \\
f(\alpha)=\frac{(2+\alpha)\left(0.886+4.64 \alpha-13.32 \alpha^{2}+14.72 \alpha^{3}-5.6 \alpha^{4}\right)}{(1-\alpha)^{\frac{3}{2}}},
\end{gathered}
$$

Table 1: Mechanical properties of materials

\begin{tabular}{c|c|c|c|c|c}
\hline \hline Material & $\begin{array}{c}\text { Yield stress } \\
\sigma_{\mathrm{y}}(\mathrm{MPa})\end{array}$ & $\begin{array}{c}0.2 \% \text { Proof } \\
\text { stress } \\
\sigma_{0.2}(\mathrm{MPa})\end{array}$ & $\begin{array}{c}\text { Tensile } \\
\text { strength } \\
\sigma_{B}(\mathrm{MPa})\end{array}$ & $\begin{array}{c}\text { Elongation } \\
\delta(\%)\end{array}$ & $\begin{array}{c}\text { Reduction } \\
\text { of area } \\
\phi(\%)\end{array}$ \\
\hline $\mathrm{A} 5052$ & - & 225 & 268 & 19.8 & 43.4 \\
\hline $\mathrm{S} 25 \mathrm{C}$ & 333 & - & 506 & 35.5 & 59.8 \\
\hline
\end{tabular}

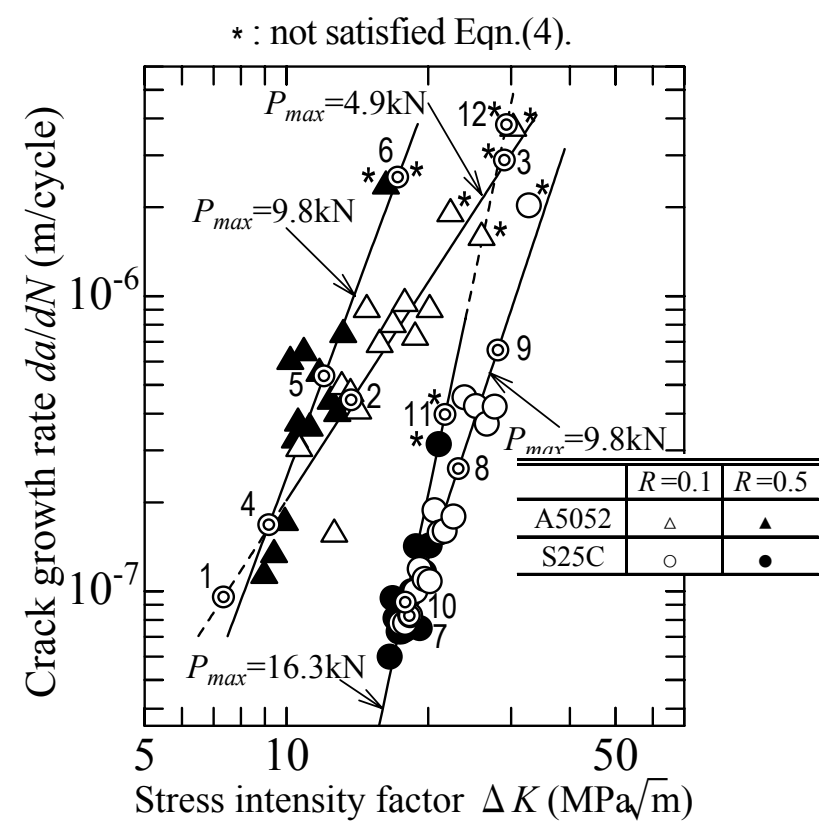

Figure 1: Relationships between stress intensity factor range $\Delta K$ and crack growth rate $d a / d N$ 
and $f(\alpha)$ is the modification coefficient depending on the specimen configuration of $\alpha=a / W$. Furthermore $\Delta P$ and $a$ mean the load range and the crack length, respectively. $\quad B$ and $W$ are the specimen thickness and width, and they are $B=16 \mathrm{~mm}$ and $W=32 \mathrm{~mm}$ for the present specimens.

When the linear fracture mechanics is applied to the fatigue crack propagation behavior, the following condition of small scale yielding [13] must be always satisfied;

$$
W-a \geq \frac{4}{\pi}\left(\frac{K_{\max }}{\sigma_{y}}\right)^{2},
$$

where $\sigma_{y}$ is the yield stress of the materials, and $K_{\max }$ is the maximum stress intensity factor calculated by substituting the maximum load $P_{\max }$ into $\Delta P$ in Eqn. (2). In this study, we have $\sigma 0.2=225 \mathrm{MPa}$ for A5052 specimen and $\sigma_{y}=333 \mathrm{MPa}$ for $\mathrm{S} 25 \mathrm{C}$ specimen as shown in Table 1. Based on calculation at every stage of the crack propagation, almost all experimental points in Figure 1 satisfy the above condition except for a few points attached the mark of “*”, which appear in the high crack growth rate region.

\subsection{Observation of Fatigue Fracture Surfaces}

Fatigue fracture surfaces were observed by means of a SLM (Scanning Laser Microscope) system. Comparing with SEM (Scanning Electronic Microscope) observations, the present SLM has some advantages such that 3-dimensional numerical data are directly obtained on real-time and these data are fundamentally convenient for the fractal analysis performed in this study.

As examples of SLM observations, micrographs of the fatigue fracture surface of A5052 aluminum alloy taken at the resolution of $\times 1250$ are shown in Figure 2 (a) and (b). For the sake of comparison, SEM micrograph of the same area is also shown in Figure 2 (c), and the striation pattern is again observed with the further high resolution as shown in Figure 2 (d). In these SLM micrographs, a bright part indicates a high position on the real surface, whereas a dark part indicates a low position. The real height (total depth) in Figure 2 (a) is $64 \mu \mathrm{m}$ and that in Figure 2 (b) is $122 \mu \mathrm{m}$, respectively. For the SLM observation with the resolution of $\times 1250$, a square area of $161.5 \mu \mathrm{m} \times 161.5 \mu \mathrm{m}$ is defined as a sample space to analyse the fracture surface irregularity. This area is replaced by a $200 \mathrm{dot} \times 200$ dot square on CRT screen. Based on the digital data thus obtained, the imaginary fracture surface was reconstructed in a 3-dimensional space as illustrated in Figure 3. Fractal analysis was performed on the geometrical

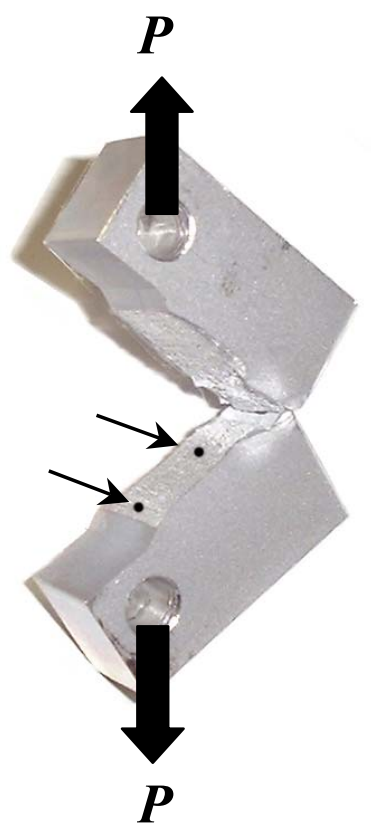

$\mathrm{A} 5052, R=0.1$, $P_{\max }=4.9 \mathrm{kN}$
Crack growth

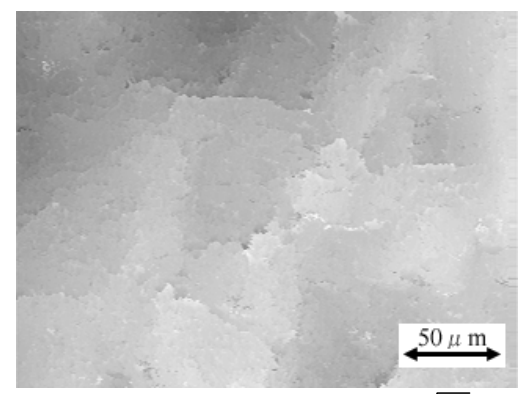

(a) $\Delta K=7.35(\mathrm{MPa} \sqrt{\mathrm{m}})$

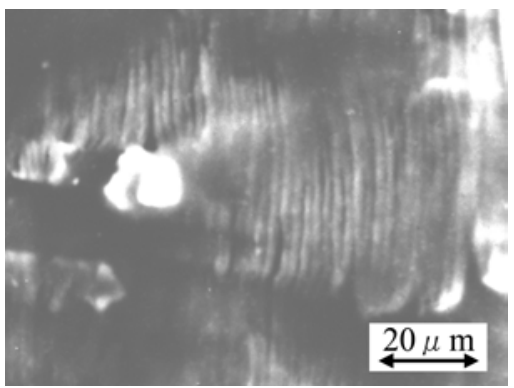

(d) Striation pattern

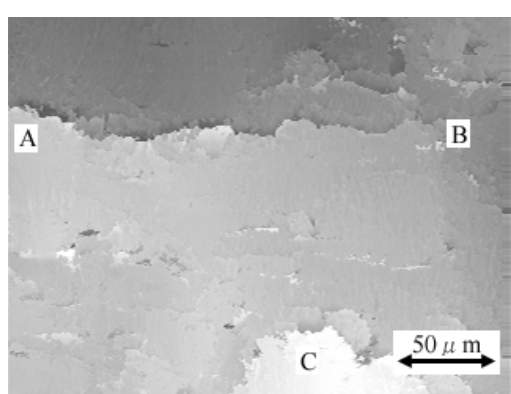

(b) $\Delta K=29.04(\mathrm{MPa} \sqrt{\mathrm{m}})$

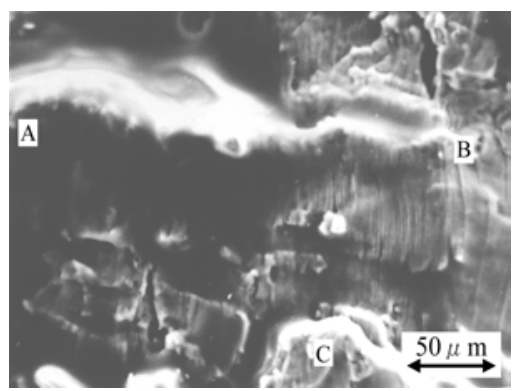

(c) $\Delta K=29.04(\mathrm{MPa} \sqrt{\mathrm{m}})$

Figure 2: (a), (b)SLM and (c), (d)SEM micrographs of fatigue fracture surface 


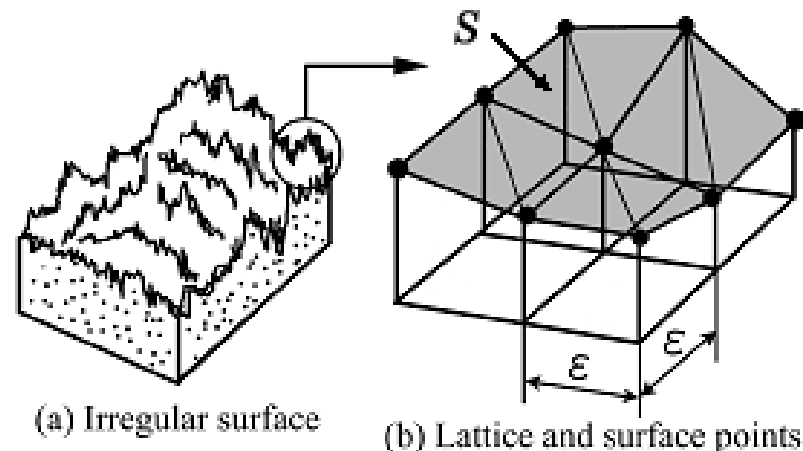

(b) Lattice and surface points

Figure 3: Reconstruction of imaginary fracture surface by multifacet surface

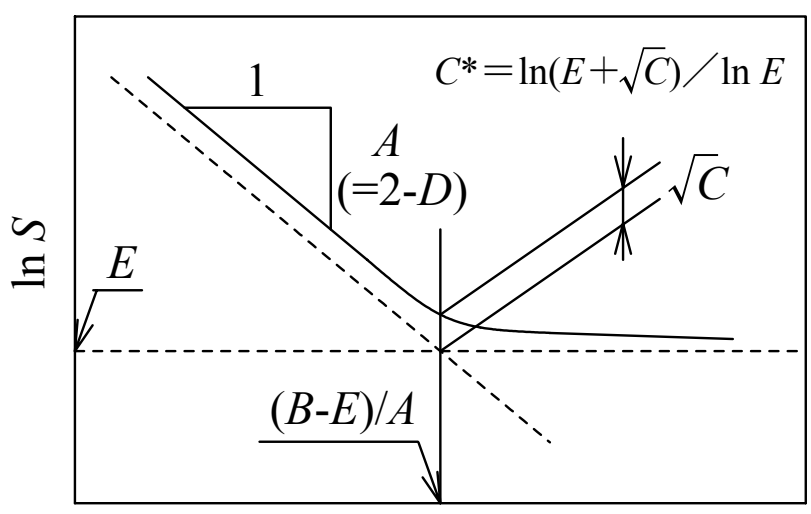

$\ln \varepsilon$

Figure 4: Schematics of $\ln \varepsilon-\ln S$ relationship

irregularity of such a imaginary fracture surface.

\section{ANALYTICAL PROCEDURE}

As reported in the earlier papers [6,7], geometrical irregularity of the tensile fracture surfaces and the mechanically finished surfaces were well evaluated by using the method of "Fractal". If a surface has the fractal nature, we have the following equation between the total area of imaginary fracture surface $S$ and the measuring unit length $\varepsilon$;

$$
\ln S=\ln F+(2-D) \ln \varepsilon,
$$

where $D$ is the fractal dimension. Thus this dimension can be calculated from the slope of the regression line for $\ln \varepsilon-\ln S$ relationship as shown in Figure 4. The value of $D$ is always in $D \geqq 2.0$, since the regression line must have a negative slope. The surface area $S$ changes depending on the unit length of $\varepsilon$, and the effect of $\varepsilon$ on the surface area $S$ is so called "Richardson effect [14]".

Usually, the linear Richardson effect is confirmed within a limited range of $\varepsilon$, so that the effect is no longer represented by the linear expression in other area of $\varepsilon$. Thus the Richardson effect in the entire region of $\varepsilon$ should be represented by a different type of expression. Accordingly, the following expression in hyperbola type [15] is proposed here;

$$
(\ln S-E)(\ln S+A \ln \varepsilon-B)=C .
$$

Meanings of all the parameters in this expression are schematically indicated in Figure 4 . Fractal dimension $D$ can be calculated from the slope of $A$ as $D=2-A$. Other parameters such as $B, C$ and $E$ are termed as "index of fracture surface nature" in this paper, since these parameters well reflect the geometrical characteristics of the fracture surface.

\section{ANALYTICAL RESULTS AND DISCUSSIONS \\ 4.1 Analytical Results and Self-similarity of Fatigue Fracture Surface}

As examples of the Richardson effect, the fracture surface area $S$ measured on A5052 alloy and S25C steel specimens was plotted as functions of the unit length $\varepsilon$ in Figure 5 (a) and (b). For the sake of comparison, the analytical results of the tensile fracture surface [6] were also plotted by using small solid symbols in Figure 5 (b). Fractal dimension and each index of fracture surface nature analysed for the respective SLM micrographs are listed in Table 2. Fractal dimensions are calculated from the slope of each regression line for the individual magnification. Each horizontal dashed line indicates the level of perfect flat surface which has $D=2.0$ for every magnification of the SLM observation. In these figures, it is found that $\ln \varepsilon-\ln S$ relationships for fatigue fracture surfaces of the objective materials are well 


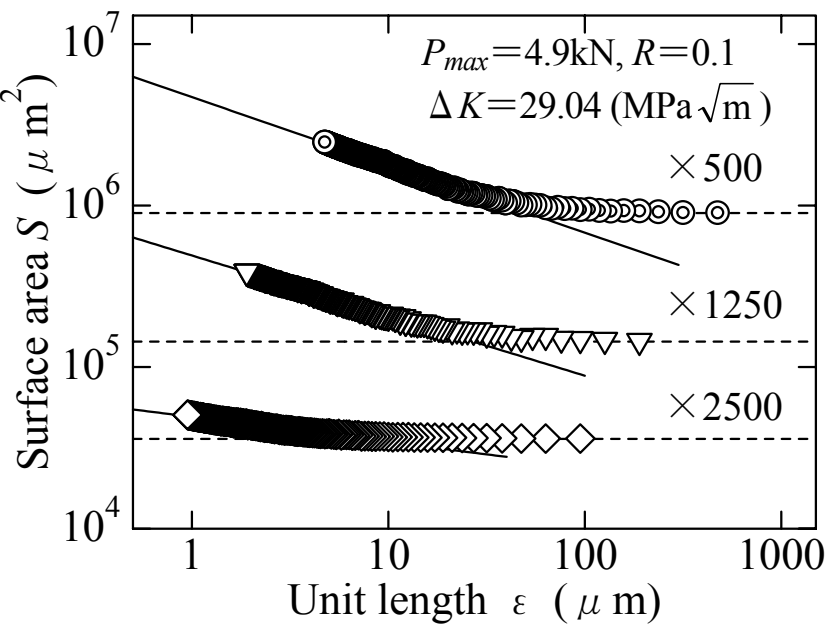

(a) A5052 aluminum alloy

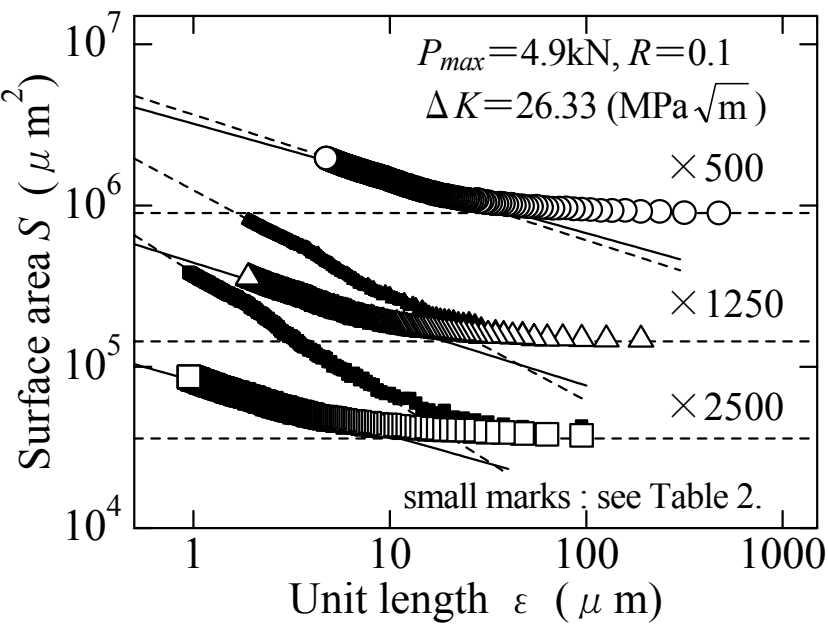

(b) S25C carbon steel

Figure 5: $\ln \varepsilon-\ln S$ relationships for A5052 aluminum alloy and S25C carbon steel under $R=0.1$

Table 2: Numerical list of analytical results $\left(P_{\max }=4.9 \mathrm{kN}\right)$

\begin{tabular}{|c|c|c|c|c|c|c|}
\hline Material & $\overline{\Delta \Delta K}$ & Mag. & Mark & $\bar{D}$ & $(B-E) / A$ & $\bar{C}^{*}$ \\
\hline \multirow{3}{*}{$\begin{array}{c}\text { A5052 } \\
\text { (Fatigue) } \\
R=0.1\end{array}$} & \multirow{3}{*}{$\begin{array}{c}29.04 \\
\mathrm{MPa} \sqrt{m}\end{array}$} & 500 & (2) & 2.42 & 51.5 & 1.13 \\
\hline & & 1250 & $\bar{\nabla}$ & 2.37 & 26.8 & 1.17 \\
\hline & & 2500 & $\diamond$ & 2.16 & 7.4 & 1.04 \\
\hline \multirow{3}{*}{$\begin{array}{c}\mathrm{S} 25 \mathrm{C} \\
\text { (Fatigue) } \\
R=0.1\end{array}$} & \multirow{3}{*}{$\begin{array}{c}26.33 \\
\operatorname{MPa} \sqrt{m}\end{array}$} & 500 & 0 & 2.34 & 42.5 & 1.12 \\
\hline & & 1250 & $\triangle$ & 2.38 & 19.1 & 1.14 \\
\hline & & 2500 & $\square$ & 2.34 & 11.1 & 1.19 \\
\hline \multirow{3}{*}{$\begin{array}{c}\text { S35C } \\
\text { (Tension) }\end{array}$} & \multirow{3}{*}{ - } & 500 & $\bullet$ & 2.39 & 36.7 & 1.11 \\
\hline & & 1250 & $\Delta$ & 2.65 & 27.1 & 1.34 \\
\hline & & 2500 & $\pi$ & 2.78 & 20.7 & 1.36 \\
\hline
\end{tabular}

represented by the expression in hyperbola type in Eqn. (6).

If the analytical object has a fractal nature, then the irregularity is well evaluated by the fractal dimension and must have characteristics of the self-similarity [1]. In Table 2, the values of $D$ are almost constant for the fracture surfaces of S25C carbon steel in wide resolution range. However, in the case of A5052 aluminum alloy, the fractal dimension tends to decrease in the higher magnification of $\times 2500$. Thus the self-similarity cannot be confirmed in the entire resolution range depending on the material. Similar trend was found on the fracture surfaces failed under $R=0.5$.

\subsection{Connection of Surface Irregularity and Fracture Mechanics}

Relationships between the stress intensity factor range $\Delta K$ and the fractal dimension $D$ were plotted in Figure 6. Each number attached to the bracket is the code number to be linked the corresponding number in Figure 1. In Figure 6 (a), it is found that the fractal dimension tends to increase with an increase of the $\Delta K$ in the lower resolution, although the value keeps constant in the higher resolution. This means that the macroscopic irregularity on the fracture surface increases with an increase of $\Delta K$, while the microscopic irregularity has no effect on $\Delta K$. On the other hand, in Figure 6 (b), the fractal dimension tends to increase as the $\Delta K$ is increased in the entire resolution range. Thus the macroscopic and the microscopic irregularities on the fracture surface of S25C increase with an increase of $\Delta K$.

Furthermore, in the relationships between the $\Delta K$ and index of fracture surface nature $(B-E) / A$, the value of $(B-E) / A$ tends to increase regardless of the resolution range as the $\Delta K$ is increased. Especially, this trend is distinct in the lower resolution range. Thus the fractal dimension and each index of fracture surface nature were successfully connected to the stress intensity factor range of $\Delta K$.

\section{CONCLUSIONS}

(1) Analytical procedure to evaluate the surface irregularity of the fatigue fracture surfaces for A5052 


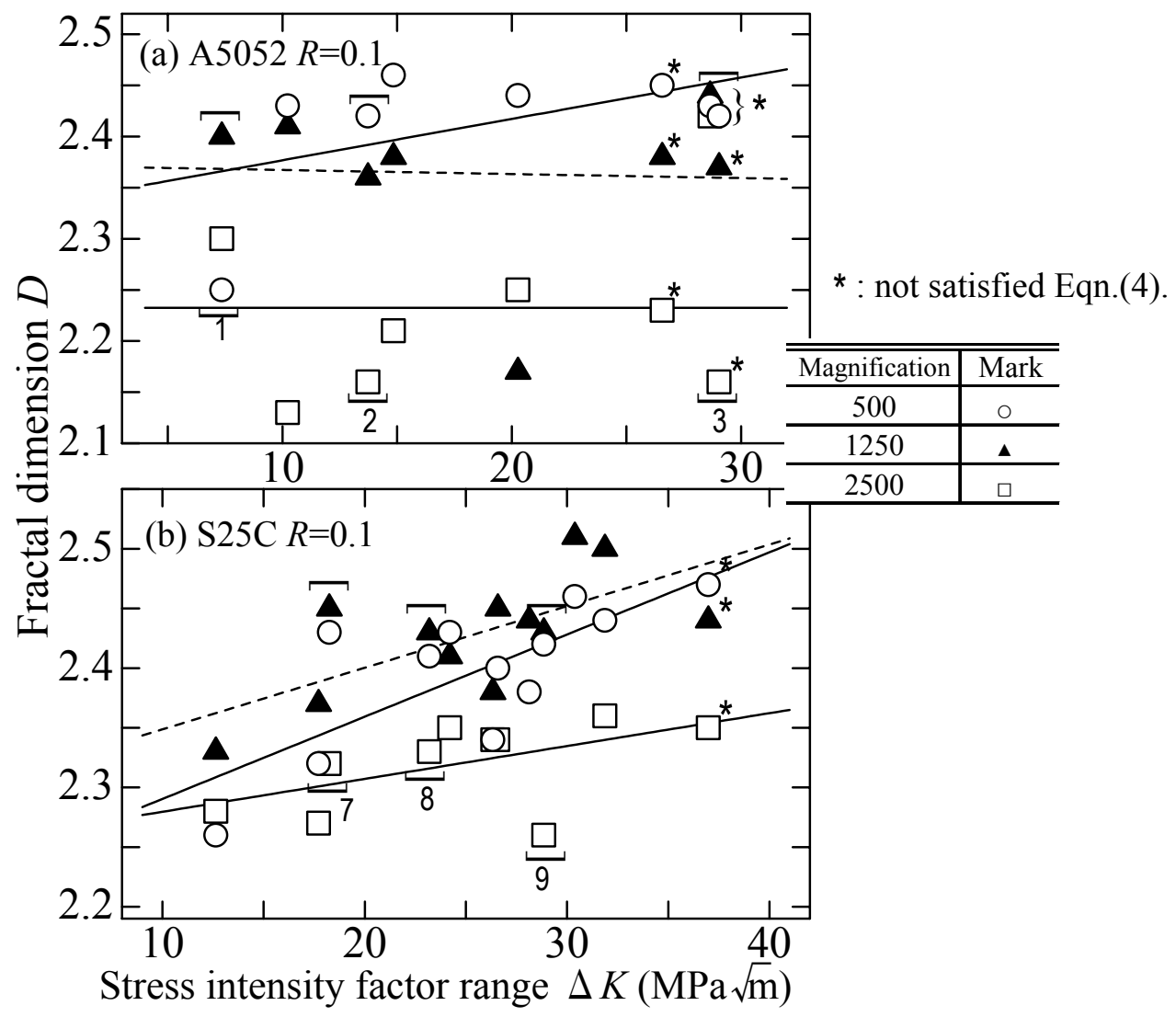

Figure 6: Relationships between stress intensity factor range $\Delta K$ and fractal dimension $D$

aluminum alloy and S25C carbon steel was developed by applying a concept of fractal and a curve fitting technique.

(2) Fatigue fracture surfaces of the present metallic materials have the fractal nature. Especially, the fracture surfaces of $\mathrm{S} 25 \mathrm{C}$ carbon steel under the stress ratios $R=0.1$ and 0.5 have the complete self-similarity in wide resolution range.

(3) In order to evaluate the geometrical irregularity of the fracture surface, one should combine the fractal dimension and additional indices termed as "index of fracture surface nature".

(4) Fractal dimension and each index of fracture surface nature were successfully connected to the stress intensity factor range $\Delta K$ giving the key parameter in the fracture mechanics approach to the crack propagation.

\section{REFFERENCES}

(1) Mandelbrot, B. B. (1983). The Fractal Geometry of Nature. W. H. Freeman and Company. 109.

(2) Bazant, Z. P. (1998). Structural Safety and Reliability. 2, 1255.

(3) Sakai, T. and Fujikawa, M. (1998). Trans. Jap. Soc. Mech. Engrs. A-64, 2271.

(4) Tanaka, M. (1998). J. Soc. Mater. Sci., Japan. 47, 169.

(5) Tsuda, M. et al. (1991). J. Soc. Mater. Sci., Japan. 40, 1066.

(6) Sakai, T. et al. (2000). Trans. Jap. Soc. Mech. Engrs. A-66, 741.

(7) Sakai, T. et al. (1998). Trans. Jap. Soc. Mech. Engrs. A-64, 1104.

(8) Srawley, J. E. (1976). Int. J. of Fract. 12, 475.

(9) Murakami, K. et al. (1987). Stress Intensity Factor Handbook. Pergamon. 1, 18.

(10) Oide, T. (1995). J. Soc. Mater. Sci., Japan. 44, 262.

(11) Masubuchi, K. (1992). Welding J. 71, 69.

(12) Paris, P. C. et al. (1963). Trans. of the ASME J. of Basic Engrs. 85, 528.

(13) ASTM E647-93. (1993). Standard Test Method for Measurement of Fatigue Crack Growth Rate.

(14) Richardson, L. F. (1961). General System Yearbook. 6, 139.

(15) Nishijima, S. (1990). Trans. Jap. Soc. Mech. Engrs. A-46, 1303. 